\title{
Unbounded Operator Algebras and DF-Spaces
}

\author{
By \\ Jean-Paul JURZAK*
}

\section{Introduction}

This paper concern the study of $*$-algebras, that is to say, algebras of operators, bounded or not, defined on a dense invariant domain $D$ of some Hilbert space H. Such objects, suitably topologized, are natural DF-spaces of analysis [5], and the aim of this paper is to present a development similar to that of $\mathbb{C}^{*}$-algebras. The work has been divided as follows. In first part, we examine properties of the domain $D$, and this leads to the analysis of the set $B(\mathfrak{D}, \mathfrak{D})$ of continuous sesquilinear forms on $\mathfrak{D} \times \mathfrak{D}$ : this space is a $\mathrm{DF}$-space, and admits a predual which is a Fréchet space. Of course, $B(\mathfrak{D}, \mathfrak{D})$ plays the role of the algebra of all bounded operators of $\mathbb{C}^{*}$-algebras. The question of normality of the positive cone of $\mathfrak{A}$ is solved for particular $*$-algebras $\mathfrak{A}$ (see part three), and its central role is described in Proposition 6. Second part describes the second dual of $\mathfrak{A}$. Third part is concerned with particular *algebras, for which we get an explicit description of the dual. The topological contents of such algebras are quite opposite to that of $\mathbb{C}^{*}$-theory. It should be pointed out that the study of positive linear forms on special $*$-algebras has been undertaken, without topology, by Shermann [11] and Woronowicz [12], and their methods are connected to our topological analysis. Theory of duality in locally convex spaces, mainly in Fréchet spaces, is essential for our study, and our bibliography in this direction is very incomplete. The reader will find main notations of this work in [9]: though [8] contains [9], its knowledge is not necessary for the understanding of this paper.

\section{Preliminaries}

In this paper, we call *-algebra, in a Hilbert space $\mathrm{H}$, an involutive algebra

Communicated by H. Araki, September 22, 1980.

* Physique-Mathématique, Faculté des Sciences Mirande, 21000 Dijon, France. 
$\mathfrak{U}$ of operators, not necessarily bounded, all defined on a domain $\mathfrak{D}$ dense in $\mathrm{H}$, with the following properties:

$1^{\circ}$ ) for $A \in \mathfrak{A}$, the adjoint operator $A^{*}$ satisfies $\operatorname{Dom} A^{*} \supset \mathfrak{D}$ and $A \mathfrak{D} \subset \mathfrak{D}$, $A^{*} \mathfrak{A} \subset \mathfrak{A}$,

$2^{\circ}$ ) for $A \in \mathfrak{U}, B \in \mathfrak{A}, x \in \mathfrak{D}$ we have

$$
(A+B) x=A x+B x, \quad(A B)(x)=A(B x) .
$$

Moreover $1 \in \mathfrak{A}$. An operator $A \in \mathfrak{A}$ is called positive (written $A \in \mathfrak{A}^{+}$, or $A \geqq 0)$ iff $(A x, x) \geqq 0$ for all $x \in \mathbb{D}$. Such an element defines canonically a vector subspace

$$
\mathfrak{N}_{A}=\left\{T \in \mathfrak{A} ; \rho_{A}(T)<+\infty\right\}
$$

where by definition

$$
\rho_{A}(T)=\sup _{x \in D} \frac{|(T x, x)|}{(A x, x)}, \quad\left(\frac{\lambda}{0}=+\infty \text { for } \lambda>0\right) .
$$

It follows that $\mathfrak{N}_{A}$ has a natural norm given by $\rho_{A}$. Clearly, $\rho_{A}$ (restricted to $\mathfrak{N}_{A}$ ) is the norm associated to the order-unit $A$ of $\mathfrak{N}_{A}$ : it is convenient to write $\|T\|_{A}$, instead of $\rho_{A}(T)$. It is immediate that subspaces $\mathfrak{N}_{A}$, for A varying in $\mathfrak{U}^{+}$, constitute an inductive system of normed spaces: by definition, the locally convex inductive limit topology of the system of normed spaces $\left(\mathfrak{N}_{A},\|\|_{A}\right)$, for $A \in \mathfrak{A}^{+}$, is called $\rho$, and the topological space so obtained is denoted simply $(\mathfrak{A}, \rho)$ : one has $\mathfrak{A}=\cup_{A \geqq 0} \mathfrak{N}_{A}$. It is easy to see that topology $\rho$ can be constructed from a sequence of subspaces $\mathfrak{N}_{A_{n}}, A_{n} \in \mathfrak{U}^{+}$for $n \in \mathbb{N}$, if and only if the positive cone $\mathfrak{U}^{+}$of $\mathfrak{U}$ admits a cofinal subset, for its natural order: equivalently, if and only if the domain $D$ is a metric space under its natural topology, defined by semi-norms $x \in \mathfrak{D} \rightarrow\|A x\|$ for $A$ varying in $\mathfrak{A}$. Algebras of this type have been called, in [9], countably dominated $*$-algebras. Since all examples met in practice are of this form, the paper will deal only with countably dominated *-algebras, denoted simply *-algebras.

Thus, a $*$-algebra $\mathfrak{A}$, endowed with $\rho$, is a separated DF-space, and the strong dual $\mathfrak{A}^{\prime}$ is a Fréchet space. If $\hat{\mathfrak{D}}$ is the completion of the metric space $\mathfrak{D}, \hat{\mathfrak{D}}$ is a Fréchet space, and obviously equal to $\cap_{A \in \mathfrak{A}} \operatorname{Dom}(\bar{A})$ (here, $\bar{A}$ stands for the closure of the operator A). It is important to note that the estimation $\rho_{A}(T)$, calculated with respect to $\mathfrak{D}$ or $\hat{\mathfrak{D}}$, defines the same numbers, so that we can assume in any theorem or proof, the equality between $\mathfrak{D}$ and $\hat{\mathfrak{D}}$.

More generally, let $\mathfrak{A}=\cup_{i \in N} \mathfrak{N}_{A_{i}}$ be a $*$-algebra, and $M$ be a vector space of 
continuous sesquilinear forms on $\mathfrak{D} \times \mathfrak{D}, M$ containing $\mathfrak{A}$ and satisfying $M=M^{*}$, where $B \rightarrow B^{*}$ is the involution of $M$ given by $B^{*}(x, y)=\overline{B(y, x)}$ (for $x, y$ in $D$ ). Here elements $T$ of $\mathfrak{A}$ are identified to forms $\beta:(x, y) \in \mathfrak{D} \times \mathfrak{D} \rightarrow \beta(x, y)=(T x, y)$ : and $\beta^{*}$ correspond therefore to element $T^{*}$ of $\mathfrak{A}$. Of course, one has $M=M_{r}$ $\oplus i M_{r}$ where $M_{r}$ is the real vector space of hermitian sesquilinear forms of M. Note that $M_{r}$ has a natural order $\leqq$, associated to the cone $M^{+}$, with

$$
M^{+}=\{\beta \in M ; \beta(x, x) \geqq 0 \quad \text { for all } x \text { of } D\} .
$$

For $\beta$ taken in $M$, there exists $M<+\infty$ and $i \in \mathbb{N}$ such that

$$
|\beta(x, y)| \leqq M\left\|A_{i} x\right\|\left\|A_{i} y\right\|
$$

hence

$$
|\beta(x, x)| \leqq M\left(A_{i}^{*} A_{i} x, x\right) .
$$

Therefore, every cofinal subset of $\mathfrak{A}^{+}$is cofinal in $M^{+}$. It follows that we can introduce, for $\beta \in M$, the quantity

$$
\sup _{x \in \mathcal{D}} \frac{|\beta(x, x)|}{\left(A_{j} x, x\right)}=\sup _{x \in \hat{\mathcal{D}}} \frac{|\beta(x, x)|}{\left(A_{j} x, x\right)} \quad\left(\frac{\lambda}{0}=+\infty \quad \text { for } \quad \lambda>0\right)
$$

which clearly defines, as precedently, a normed space $\left(\mathscr{N}_{A_{j}},\|\|_{A_{j}}\right)$ with order unit $A_{j}$ and a natural topology $\rho$ on $M$ given by

$$
(M, \rho)=\underset{j}{\operatorname{ind} \lim }\left(\mathscr{N}_{A_{j}},\|\|_{A_{j}}\right) .
$$

One check that linear forms $\omega_{x, x}(x$ being in $\mathfrak{D})$ on $M$, defined by

$$
M \longrightarrow \omega_{x, x}(\beta)=\beta(x, x)
$$

are continuous, hence $(M, \rho)$ is separated. Moreover, since every simply bounded set of sesquilinear continuous forms on $\mathfrak{D} \times \mathfrak{D}$ is equicontinuous, a fundamental system of bounded sets of $(M, \rho)$ is given by intervals $[-\Delta, \Delta]$, with $\Delta$ varying in $M^{+}$(or $\mathfrak{A}^{+}$). We recall that $[\mathrm{A}, \mathrm{B}]$, for $\mathrm{A}, \mathrm{B}$ in $M^{+}$, denotes the set $\left(A+M^{+}\right) \cap\left(B-M^{+}\right)$.

Of course, one can endow $\mathfrak{A}$ or $M$ with many other topologies. For example, we can put on $M$ the topology of bibounded convergence (resp. bicompact convergence...) which is, taking in account the polarization equality, given by semi-norms

$$
T \longrightarrow \sup _{x \in B}|(T x, x)|
$$

for $B$ varying in the family of bounded sets (resp. compact...) of $\mathfrak{A}$. It is trivial 
that bounded sets for these topologies agree with bounded sets of $(M, \rho)$. But, these topologies are a priori not sufficiently fine, and it is not known, in general, if vector spaces so obtained are quasi-barreled, or if their strong dual is complete. Topology $\rho$ introduced is exactly the bornological space associated with $\mathfrak{A}$ endowed with bibounded topology, and invariance of $\rho$ under isomorphisms, as many other properties [9], make interest in this approach.

A technical topology of some interest, in the study of a given *-algebra $\mathfrak{A}$, can be obtained by introducing estimations of the form $\sup _{x \in \mathfrak{D}}\|T x\| /\|A x\|$, with $T, A$ in $\mathfrak{A}$, and usual convention $\lambda / 0=+\infty$ for $\lambda>0$ : such a procedure leads, for every $A$ of $\mathfrak{A}$, to a natural definition of the normed space $\left(\mathfrak{M}_{A},\|\| \|_{A}\right)$, hence to a topology, called $\lambda$ in [9], equal by definition to the locally convex inductive limit topology of the system of normed spaces $\left(\mathfrak{M}_{A},\|\|_{A}, A \in \mathfrak{L}\right)$. Continuity of multiplication $(S, T) \in \mathfrak{U} \times \mathfrak{U} \rightarrow S T \in \mathfrak{U}$ is equivalent to the equality of topologies $\lambda$ and $\rho,[8]$, thus presence of $\lambda$ is indispensable only for certain *-algebras.

\section{$\S 1$. Generalities}

Let $\mathfrak{D}$ be the domain of a given *-algebra $\mathfrak{A}=\cup_{i \in N} \mathfrak{N}_{A_{i}}$, and $H$ be the Hilbert space completion of the prehilbert space $D$. As mentioned in introduction, we can assume, without loss of generality, the topological space $\mathfrak{D}$ equal to its completion $\hat{\mathfrak{D}}$ : it follows that $\mathfrak{D}$ is a Fréchet space, under semi-norms $x \in \mathfrak{D} \rightarrow\left\|A_{i} x\right\|($ for $i \in \mathbb{N})$.

Proposition 1. If $M$ is a bounded subset of the strong dual $\mathfrak{D}^{\prime}$ of $\mathfrak{D}$, then there exists an integer $i$ and a real number $\alpha$, such that $M$ is included in the set of linear forms $f$ on $\mathfrak{D}$, of type $f(x)=\left(A_{i} x, y\right)$, with y varying in $H$, with norm smaller than $\alpha$.

In particular, if the Hilbert space $H$ is separable, the strong dual $\mathfrak{D}^{\prime}$ is separable, therefore bornological. Converse of the proposition is trivial.

Proof. Since $D$ is a Fréchet space, $M$ is an equicontinuous set, therefore there exists $i \in N$ and $\alpha>0$ such that

$$
|f(x)| \leqq \alpha\left\|A_{i} x\right\|
$$

for all $x$ of $\mathfrak{D}$ and $f$ of $M$. We can assume $A_{i}^{*} A_{i} \geqq \mathrm{Id}$, and we get

$$
|\tilde{f}(u)| \leqq \alpha\|u\| \quad \text { for } \quad u \in A_{i}(\mathfrak{D})
$$


having introduced $\tilde{f}$ by the relation

$$
\tilde{f}\left(A_{i} x\right)=f(x)
$$

which leads immediately to the result. Let us now assume that $H$ is separable, and denote $\left\{y_{i}\right\}$ a sequence of vectors dense in the Hilbert space $H$. Linear forms $\left(A_{i} \cdot y_{j}\right)$, for $i$ and $j$ varying in $\mathbb{N}$, are dense in the strong dual $\mathfrak{D}^{\prime}$, because one has, for a given $y$ of $H$

$$
\sup _{x \in B}\left|\left(A_{i} x, y\right)-\left(A_{i} x, y_{j}\right)\right| \leqq\left\|y-y_{j}\right\| \sup _{x \in B}\left\|A_{i} x\right\|
$$

which tends to zero when $y_{j}$ tends to $y$ in $H$, for all bounded sets $B$ of $D$.

Proposition 2. Let $D$ be the domain of a *-algebra 2 . Then, $D$ is a Schwartz space (resp. a nuclear space) if and only if $\mathfrak{A}$ can be written $\mathfrak{U}=$ $\cup_{i \in \mathbb{N}} \mathfrak{M}_{A_{i}}$ (or $\left.\mathfrak{A}=\cup_{j \in \boldsymbol{N}} \mathscr{M}_{A_{j}}\right), A_{i} A_{i+1}^{-1}$ being, for every $i \in \mathbb{N}$, a compact operator (resp. a nuclear operator) acting in the Hilbert space $H$.

Proof. Every $A$ of $\mathfrak{A}$ defines a semi-norm $p_{A}$ on $\mathfrak{D}$ by $p_{A}(x)=\|A x\|$, hence defines the disked neighborhood $U=\{x \in \mathfrak{D} ;\|A x\| \leqq 1\}$, the normed space $\mathfrak{D}_{U}$ with "unit ball $U$ ", and $\hat{\mathfrak{D}}_{U}$ the Banach space completion of $\mathfrak{D}_{U}$. When $A^{*} A \geqq \mathrm{Id}, \mathfrak{D}_{U}$ (resp. $\hat{\mathfrak{D}}_{U}$ ) can be identified with a subspace (resp. a closed subspace) of $H$, by the map

$$
x \in \mathfrak{D}_{U} \longrightarrow A x \in H
$$

Taking $B$ another element of $\mathfrak{A}$, and denoting by $V$ the corresponding neighborhood in $\mathfrak{D}$, we see that $V \subset U$ if and only if $\|A x\| \leqq\|B x\|$ for all $x \in \mathfrak{D}$. Thus, the canonical map from $\hat{\mathfrak{D}}_{V}$ into $\hat{\mathfrak{D}}_{U}$ corresponds to the operator $A B^{-1}$, acting in the Hilbert space $H$ (in fact, $A B^{-1}$ is defined on $B(D)=\overline{B(D)}$ and is extended to whole $H$ by choosing its value equal to zero on the orthogonal complement of $B(D))$. Now, the possibility of choosing $V$ arbitrarily small means that we can take, for every given $i \in \mathbb{N}$, an element $B$ of $\mathfrak{A}$ such that $\|B x\| \geqq\left\|A_{i} x\right\|$ for all $x \in \mathcal{D}$. Therefore, the existence of operators $A_{i}$ follows from definitions of nuclear and Schwartz spaces [5], [6].

Proposition 3. Under its natural topology, D is a reflexive Fréchet space, and this property holds for every quotient and every closed subspace of a finite product of $\mathfrak{D}$. Bounded subsets of $\mathfrak{D}$ are relatively weakly compact subsets.

To be more precise, the proof of the last proposition shows that $D$ is isomorphic to a topological vector subspace of a topological vector product of a 
family of Hilbert spaces. The affirmation relative to a finite product is obtained by finite product of copies of $\mathfrak{D}$ and weak compactness follows from reflexivity.

Using now a criterion in [6] page 70 remarque 2 , we deduce

Theorem 1. Every bounded subset of the Fréchet space $\mathfrak{D} \hat{\otimes} \mathfrak{D}$ is contained in the canonical image of the unit ball of a space $\mathfrak{D}_{A} \widehat{\otimes} \mathfrak{D}_{A}$ where $A$ is a bounded closed disk if $\mathfrak{D}$.

Here, $\hat{\otimes}$ stands for the projective tensor product. Thus, from [6], we get

Corollary 1 . The set $B(\mathfrak{D}, \mathfrak{D})$ of continuous sesquilinear forms on $\mathfrak{D}$ $\times D$ endowed with bibounded topology, is the strong dual of the Fréchet space $\mathfrak{D} \hat{\otimes} \mathfrak{D}$.

The natural duality $\langle\mathfrak{D} \hat{\otimes} \mathfrak{D}, B(\mathfrak{D}, \mathfrak{D})\rangle$ leads us to introduce the following.

Definition 1. We call the weak topology on $\mathfrak{A}$ (resp. the $\sigma$-weak topology), relative to $\mathfrak{D} \otimes \mathfrak{D}$, the topology on $\mathfrak{A}$ induced by $\sigma(B(\mathfrak{D}, \mathfrak{D}), \mathfrak{D} \otimes \mathfrak{D}$ ) (resp. by $\sigma(B(\mathfrak{D}, \mathfrak{D}), \mathfrak{D} \hat{\otimes} \mathfrak{D}), \mathfrak{A}$ being an arbitrary subset of $B(\mathfrak{D}, \mathfrak{D})$.

In other words, the weak topology on a linear space $\mathfrak{A}$ is the topology associated to semi-norms

$$
T \longrightarrow|(T x, y)|
$$

for $x$ and $y$ varying in $\mathfrak{D}=\hat{\mathfrak{D}}$. Clearly, the dual of $\mathfrak{A}$ for this topology is the linear span of the $\omega_{x, y}$, with $x, y$ belonging to $\mathfrak{D}$. The $\sigma$-weak topology on $\mathfrak{I}$ is the topology defined by semi-norms

$$
T \longrightarrow\left|\sum_{j=1}^{\infty}\left(T x_{j}, x_{j}\right)\right|
$$

or

$$
T \longrightarrow\left|\sum_{j=1}^{\infty}\left(T x_{j}, y_{j}\right)\right|,
$$

$\left(x_{j}\right)_{j \in N}\left(\right.$ resp. $\left.\left(y_{j}\right)_{j \in N}\right)$ being any $\sigma$-convergent sequence of $\mathfrak{D}$ (i.e. $\sum_{j=1}^{\infty} A\left(x_{j}, x_{j}\right)$ $<+\infty$ for every $A$ of some cofinal set of $B^{+}(\mathfrak{D}, \mathfrak{D})$, see [8]): every element $u$ of $\mathfrak{D} \widehat{\otimes} \mathfrak{D}$ is indeed the sum of an absolutely convergent series $\sum_{i=1}^{\infty} \lambda_{i} x_{i} \otimes y_{i}$, with $\sum_{i=1}^{\infty}\left|\lambda_{i}\right|<+\infty$ and $\left(x_{i}\right)_{i \in \boldsymbol{N}},\left(y_{i}\right)_{i \in \boldsymbol{N}}$ sequences of elements in $\mathcal{D}$ converging to zero, thus by polarization, $u$ is a finite sum of elements of the form $v=\sum_{j=1}^{\infty} \mu_{j} \bar{z}_{j} \otimes z_{j}$, with $\mu_{j} \geqq 0$ for all $j, \sum_{j=1}^{\infty} \mu_{j}<+\infty$ and $\left(z_{j}\right)_{j \in N}$ a sequence tending to zero in $\mathfrak{D}$. Since, for $T \in B(\mathfrak{D}, \mathfrak{D})$ one has

$$
\left\langle T, \sum_{j=1}^{\infty} \mu_{j} z_{j} \otimes z_{j}\right\rangle=\sum_{j=1}^{\infty} \mu_{j} T\left(z_{j}, z_{j}\right),
$$


we get semi-norms mentioned. It is clear that the dual of $\mathfrak{U}$ for $\sigma$-weak topology is the set of linear forms $\sum_{j=1}^{\infty} \omega_{x_{j}, y_{j}},\left(x_{j}\right)_{j \in \boldsymbol{N}}$ and $\left(y_{j}\right)_{j \in N} \sigma$-convergent as indicated.

In particular, the dual of ( $\mathfrak{A}$, weak) (resp. ( $\mathfrak{A}, \sigma$-weak)) is contained in the dual of $(\mathfrak{2}, \rho)$, and one can check that $\sigma$-weakly continuous linear forms on $\mathfrak{A}$ belong to the closure, relative to the topology of the strong dual of ( $\mathfrak{T}, \rho$ ), of the dual of ( $\mathfrak{A}$, weak). By Ascoli theorem, the weak topology and the $\sigma$-weak topology on $\mathfrak{A}$ agree on bounded sets of $(\mathfrak{A}, \rho)$.

It should be pointed out that any $*$-algebra $\mathfrak{A}$ can be naturally considered as a subspace of the strong dual of $\mathfrak{D} \hat{\otimes} H$, and, in this point of view, the weak topology and the $\sigma$-weak topology, relative to $\mathfrak{D} \otimes H$, make sense. These notions are of interest only when $\lambda$ agree with $\rho$ (see [8]).

Proposition 4. Any continuous (resp. equicontinuous set of) linear forms on $\mathfrak{D} \hat{\otimes} \mathfrak{D}$ corresponds, under trivial identification, to sesquilinear forms $\beta$ on $\mathfrak{D} \times \mathfrak{D}$ of the form $\beta(x, y)=\left(B A_{i} x, A_{i} y\right)$ (with $\left.x, y \in \mathfrak{D}\right)$, with $A_{i}$ suitably chosen in the positive cone of $\mathfrak{H}$, and $B$ being an $y$ (resp. $B$ varying in a set of uniformly) bounded operators acting in the Hilbert space $H$.

Recall that the set $B(\mathfrak{D}, \mathfrak{D})$ is ordered by the cone $B^{+}(\mathfrak{D}, \mathfrak{D})$ of positive sesquilinear forms on $\mathfrak{D} \times \mathfrak{D}$ : and every cofinal subset of the positive cone of $\mathscr{Q}$ is cofinal in $B^{+}(\mathfrak{D}, \mathfrak{D})$.

Proof. If $\beta$ is a continuous sesquilinear form on $\mathfrak{D} \times \mathfrak{D}$, we get, from continuity, a relation of the form

$$
|\beta(x, y)| \leqq M\left\|A_{i} x\right\|\left\|A_{i} y\right\| \quad x, y \in \mathfrak{D}
$$

where $M$ is a finite number, and $A_{i}$ correctly chosen. Putting $\tilde{\beta}\left(A_{i} x, A_{i} y\right)$ $=\beta(x, y)$ we obtain a continuous sesquilinear linear form on $A_{i}(\mathfrak{D}) \times A_{i}(\mathfrak{D})$ satisfying

$$
|\tilde{\beta}(u, v)| \leqq M\|u\|\|v\| .
$$

It is therefore trivial that $\tilde{\beta}(u, v)=(B u, v)$ for some bounded operator $B$, acting in the Hilbert space $H$ and choosen equal to zero on the orthogonal complement of $A_{i}(\mathfrak{D})$. The argument holds for an equicontinuous set of $(\mathfrak{D} \hat{\otimes} \mathfrak{D})^{\prime}$, proving our assertion.

Proposition 5. Let $\mathfrak{A}=\cup_{i \in N} \mathfrak{N}_{A_{i}}$ be a subspace of $B(\mathfrak{D}, \mathfrak{D})$, stable under involution and containing a cofinal subset of the positive cone of $B(D, D)$. 
Then $\mathfrak{A}$ is $\sigma$-weakly closed if and only if intervals $\left[-A_{i}, A_{i}\right]$ of $\mathfrak{A}$ are $\sigma$-weakly (or weakly) compacts, for all $i \in \mathbb{N}$. When it is the case, every uniformly bounded increasing directed set of hermitians elements of $\mathfrak{A}$ converges to its least upper bound for weak or $\sigma$-weak topology.

Proof. One checks easily that the involution $\beta \rightarrow \beta^{*}$ of $B(\mathfrak{D}, \mathfrak{D})$ is weakly and $\sigma$-weakly continuous. Thus it is sufficient to work on the set of hermitian elements of $\mathfrak{H}$, denoted always $\mathfrak{U}_{\mathbb{R}}$. The intervals $\left[-A_{i}, A_{i}\right]$, and their homotheties, relatively to order of $B(\mathfrak{D}, \mathfrak{D})$, with $i$ varying in the set of integers, form a fundamental system of bounded sets of $(B(\mathfrak{D}, \mathfrak{D}), \rho)$ and are convex disked equicontinuous sets. These sets are also weakly and $\sigma$-weakly closed, since one has

$$
\begin{aligned}
{\left[-A_{i}, A_{i}\right] } & =\left\{\beta \in B(\mathfrak{D}, \mathfrak{D}) ;|\beta(x, x)| \leqq A_{i}(x, x) \text { for all } x \in \mathfrak{D}\right\} \\
& =\bigcap_{x \in \mathfrak{D}}\left\{\beta \in B(\mathfrak{D}, \mathfrak{D}) ;\left\langle\frac{x}{\sqrt{A_{i}(x, x)}} \otimes \frac{x}{\sqrt{A_{i}(x, x)}}, \beta\right\rangle \leqq 1\right\} .
\end{aligned}
$$

Thus, by Ascoli theorem, these intervals are $\sigma$-weakly compact (or weakly compact, $\mathfrak{D} \otimes \mathfrak{D}$ being dense in $\mathfrak{D} \hat{\otimes} \mathfrak{D})$, hence are exactly polars of neighborhood of zero, by bipolar theorem. Therefore, the proposition follows from a theorem of Banach-Dieudonné, characterizing weakly closed subspaces of dual of Fréchet spaces. Assertion relative to uniformly bounded increasing directed set follows from preceeding compactness.

An important notion, essential for our analysis, is summing up in the following

Definition 2. Let $\mathfrak{A}$ be an ordered vector space with positive cone $\mathfrak{A}^{+}$. A vector topology $\mathscr{C}$ on $\mathfrak{A}$ is said to be order-convex if it admits a base of neighborhoods of the zero consisting of order-convex sets. When it is so, the cone $\mathfrak{H}^{+}$is called a normal cone for $(\mathfrak{A}, \mathscr{C})$.

Recall that a subset $A$ of $\mathfrak{N}$ is order-convex, if $\left[a_{1}, a_{2}\right] \subset A$ whenever $a_{1}$, $a_{2} \in A$, and $a_{1} \leqq a_{2}$. Since this definition is mainly adapted for real ordered vector spaces, we will from now on turn our attention on the real vector space of symmetric elements of a $*$-algebra $\mathfrak{A}$, or, more generally on the set $\mathfrak{A}_{\mathscr{R}}$ of symmetric elements of a subspace $\mathfrak{A}$ of $B(\mathfrak{D}, \mathfrak{D})$, stable under involution and containing a cofinal subset of the positive cone of $B(\mathfrak{D}, \mathfrak{D})$. Such an $\mathfrak{A}$ splits in the topological direct sum $\mathfrak{U}=\mathfrak{A}_{\mathscr{R}} \oplus i \mathfrak{U}_{\mathscr{R}}$ under $\rho$, and it follows that the study of $\mathfrak{U}_{\mathscr{R}}$ and $\mathfrak{A}$ are equivalent. 
This question of normality of such an $\mathfrak{A}$ has not been solved in its great generality. Only positive answers has been obtained in particular cases [8], [1] and, due to this fact, normality of $\mathfrak{A}^{+}$will be required in the next propositions. Let us note that normality is preserved under bijective positive linear maps, since such applications preserve $\rho$-norms (the same proof as in [9]).

Proposition 6. Let $\mathfrak{H}, \mathfrak{B}$ be subspaces of $B(\mathfrak{D}, \mathfrak{D})$, stable under involution and containing a cofinal subset of the positive cone of $B(\mathfrak{D}, \mathfrak{D})$ with $\mathfrak{A} \subset \mathfrak{B}$. Denote the embedding from $\mathfrak{A}$ to $\mathfrak{B}$ by $i$. If the positive cone $\mathfrak{A}^{+}$is normal under $\rho$, then the following properties holds:

$1^{\circ}$ the space $(\mathfrak{B}, \rho)$ induces on $\mathfrak{A}$ the topology $(\mathfrak{A}, \rho)$,

$2^{\circ}$ the transposed mapping ${ }^{t} i$ of the strong dual $\mathfrak{B}^{\prime}$ of $\mathfrak{B}$ into the strong dual $\mathfrak{A}^{\prime}$ of $\mathfrak{A}$ is a surjective homomorphism of Fréchet spaces. Every bounded subset of strong $\mathfrak{Q}^{\prime}$ is contained in the canonical image of a bounded subset of strong $\mathfrak{B}^{\prime}$.

$3^{\circ}$ For every sequence $y_{j}$ in $\mathfrak{A}^{\prime}$ tending to zero, there exists a sequence $x_{j}$ in $\mathfrak{B}^{\prime}$ tending to zero such that ${ }^{t} i\left(x_{j}\right)=y_{j}$ for every $j \in \mathbb{N}$,

$4^{\circ}$ the strong bidual $\mathfrak{B}^{\prime \prime}$ induces on $\mathfrak{Q}^{\prime \prime}$ the topology of strong bidual of $\mathfrak{I}^{\prime \prime}$. The positive cone of $\mathfrak{I}^{\prime \prime}$, the bipolar of $\mathfrak{A}^{+}$, is generating in $\mathfrak{I}^{\prime \prime}$ and normal.

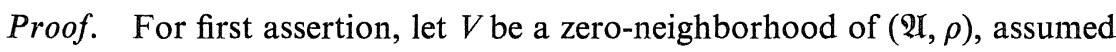
convex, disked and order-convex for the order of $\mathfrak{A}$. Denote $[V]$ the saturated hull of $V$ relatively to the order of $\mathfrak{B}:[V]$ consists of elements $T$ of $\mathfrak{B}$ for which there exists $S_{1}$ and $S_{2}$ in $V$ with $S_{1} \leqq T \leqq S_{2}$. It follows that $[V]$ is convex, disked, and satisfies $[V] \cap \mathfrak{A}=V$. Therefore it remains to show that $[V]$ is a zero-neighborhood of $(\mathfrak{B}, \rho)$. Put $\mathfrak{A}=\cup_{j} \mathfrak{N}_{A_{j}}, V_{j}=V \cap \mathfrak{N}_{A_{j}}$, and $\mathfrak{B}=\cup_{j} \mathscr{N}_{A_{j}}$. Clearly $V_{j}$ is convex, disked and saturated for the order of $\mathfrak{A}$ and the saturated hull $W_{j}$ of $V_{j}$ in $\mathscr{N}_{A_{j}}$ coincides with the saturated hull of $V_{j}$ in $\mathfrak{B}$. Since $V_{j}$ is a zero-neighborhood of the normed space $\mathfrak{N}_{A_{j}}$, it contains a suitable homothetic of $A_{j}$, implying that $W_{j}$ is a zero-neighborhood of $\mathscr{N}_{A_{j}}$. From relation [V] $\cap \mathscr{N}_{A_{j}} \supset W_{j}$, we get that $V$ is a zero-neighborhood of $(\mathfrak{B}, \rho)$, showing identity of topologies on $\mathfrak{U}$.

From normality and Proposition 1.1 [8], every positive bounded subset of $\mathfrak{U}^{\prime}$ is contained in the image of a bounded subset of $\mathfrak{B}^{\prime}$ and therefore the second assertion follows from normality of $\mathfrak{A}^{+}$and a classical theorem of homomorphisms. From quasi-normability of $\rho$-topology, we deduce lifting of strongly convergent sequences of linear forms on $(\mathfrak{I}, \rho)$. Since bounded sets of $\mathfrak{B}^{\prime}$ 
comes from bounded sets of $\mathfrak{U}^{\prime}$, by polarity, we get that the dual $\mathfrak{B}^{\prime \prime}$ of $\mathfrak{B}^{\prime}$ induces on $\mathfrak{U}^{\prime \prime}$ the strong topology of $\mathfrak{U}^{\prime \prime}$. Seminorms of the strong dual $\mathfrak{U}^{\prime}$ of $\mathfrak{U}$ have been explicited in [8] implying immediately that the positive cone of $\mathfrak{U}^{\prime}$ is normal: it follows that the positive cone of $\mathfrak{A}^{\prime \prime}$ is generating. Now, the topology of $\mathfrak{Q}^{\prime \prime}$ is also the topology of uniform convergence on bounded positive subsets of $\mathfrak{U}^{\prime}$ which leads to normality of the positive cone of $\mathfrak{U}^{\prime \prime}$.

\section{§ 2. Second Dual}

Let $\mathfrak{A}$ be a $*$-algebra or any subspace (denoted also by $\mathfrak{A}$ ), stable under involution and containing the given $*$-algebra. Endowed with bibounded topology, $\mathfrak{A}$ is a topological vector subspace of the strong dual of $\mathfrak{D} \hat{\otimes} \mathfrak{D}$ by Corollary 1. By Grothendieck analysis, we know that in general a subspace of strong dual of Fréchet space does not inherit properties of the dual itself. As a particular example, let us considerer the case where $\mathfrak{A}$ is a weakly closed subspace of $B(\mathfrak{D}, \mathfrak{D})$, in the duality $\langle\mathfrak{D} \hat{\otimes} \mathfrak{D}, B(\mathfrak{D}, \mathfrak{D})\rangle$ (with vocabular of Definition $1, \mathfrak{A}$ is an ultraweakly closed subspace of $B(\mathfrak{D}, \mathfrak{D})$ ). The bipolar theorem tell us that $\mathfrak{U}$ is, as vector space, the dual of a quotient $P$ of $\mathfrak{D} \hat{\otimes} \mathfrak{D}$, therefore $\mathfrak{A}$ is algebraically the dual of some Fréchet space $P$. However, the strong topology of $\mathfrak{A}$ (i.e. the topology of uniform convergence on bounded subsets of $P$ ) is finer than bibounded topology of $\mathfrak{A}$ : and equality holds if and only if every bounded subset of $P$ is contained in the closure of a canonical image of a bounded subset of $\mathfrak{D} \widehat{\otimes} D$. Bounded subsets of the strong dual of $P$ are equicontinuous subsets, therefore by Hahn-Banach theorem, are equicontinuous subsets of $\left((\mathfrak{D} \hat{\otimes} \mathfrak{D})^{\prime}\right)$, contained in $\mathfrak{Y}$. In short, bounded subsets of $\mathfrak{A}$, for strong topology, or bibounded topology, or $\rho$-topology coincide, and a fundamental system of such sets can be obtained by taking order intervals $[-A, A]$, A varying in $\mathfrak{A}^{+}$(since one has $\mathfrak{A}=\cup_{i \in N} \mathfrak{N}_{A_{i}}$, with $A_{i} \geqq 0$ for all $i$ ). In fact, topology $\rho$ being bornological, is the finest of these there topologies.

In order to clarify this too large generality, we will assume that the domain $\mathfrak{D}$ is, under its Fréchet topology, a quasi-normable space (a notion due to Grothendieck [5]). Since all Fréchet spaces met in analysis are of this type, this can be considered as a reasonable assumption, whose interest is that it implies properties of $\mathfrak{A}$ invariant under any isomorphism of the space $\mathfrak{A}$. In fact, for some next propositions, it suffices to know (or to show) that $B(\mathfrak{D}, \mathfrak{D})$ is, under bibounded topology, a bornological space, a property weaker than 
quasi-normability of $\mathfrak{D}$. However, questions relative to second dual of $\mathfrak{A}$ require deeper informations, deduced here from quasi-normability of $\mathfrak{D}$.

Definition 3. The Fréchet space $\mathfrak{D}$ is quasi-normable iff for every equicontinuous disk $A$ in $\mathfrak{D}^{\prime}$ there exists an equicontinuous disk $B \supset A$ such that on $A$ the topologies induced by strong $\mathfrak{D}^{\prime}$ and by $\mathfrak{D}_{B}^{\prime}$ are the same.

Note that Schwartz spaces, hence nuclear spaces, considered in Section 3 satisfy quasi-normability, and these particular situations have been already met in [8] with examples chosen among enveloping algebras.

Proposition 7. Let $\mathfrak{U}$ be a $*$-algebra with quasi-normable domain $\mathfrak{D}$ and $\overline{\mathfrak{A}}$ be the $\sigma$-weak closure of $\mathfrak{U}$. Then

$1^{\circ} \overline{\mathfrak{A}}$ is as a vector space, the strong dual of a Fréchet space,

$2^{\circ}$ the strong topology of $\overline{\mathfrak{A}}$ is equal to $\rho$-topology.

Proof. Introducing the duality $\langle\mathfrak{D} \hat{\otimes} \mathfrak{D}, B(\mathfrak{D}, \mathfrak{D})\rangle$, we see that $\overline{\mathfrak{I}}$ is vectorially the dual of the Fréchet space $\mathfrak{D} \otimes \mathfrak{D} / \mathfrak{A}^{\circ}$ where $\mathfrak{A}^{\circ}$ is the polar of $\mathfrak{A}$. Since quasi-normability is closed under projective tensor products and formation of quotients, the strong dual of $\mathfrak{D} \hat{\otimes} \mathfrak{D} / \mathfrak{H}^{\circ}$ is bornological, which implies the second assertion.

Theorem 2. Let $\mathfrak{A}$ be a subspace of $B(\mathfrak{D}, \mathfrak{D})$, stable under involution and containing a cofinal subset of the positive cone of $B(D, D)$. Assume that $D$ is quasi-normable. Then

$1^{\circ}$ the bibounded topology and the $\rho$-topology on $\mathfrak{A}$ agree on bounded subsets of $\mathfrak{H}$,

$2^{\circ}$ the space $\mathfrak{A}$, endowed with $\rho$, satisfies the strict condition of Mackey convergence. In particular, the second dual $\mathfrak{U}^{\prime \prime}$ of $\mathfrak{U}$ is a bornological DFspace for its strong topology.

Thus, if $\mathfrak{B}$ is a subspace of $B(\mathfrak{D}, \mathfrak{D})$, with $\mathfrak{B}=\mathfrak{B}^{*}$ and $\mathfrak{B} \subset \mathfrak{U}$, we get that $(\mathfrak{B}, \rho)$ agrees with $(\mathfrak{H}, \rho)$ on any bounded subset of $\mathfrak{P}$. The second assertion implies the weaker following statement: if $T_{n}$ is a sequence of elements of $\mathscr{A}$ $=\cup_{i} \mathfrak{N}_{A_{i}}$, converging to $T$ in $\mathfrak{A}$ (endowed with $\rho$-topology), there exists some integer $i$ and a sequence $\varepsilon(n)$ of real numbers, tending to zero such that

$$
\left|\left(\left(T_{n}-T\right) x, x\right)\right| \leqq \varepsilon(n)\left(A_{i} x, x\right)
$$

for all $x$ of $\mathfrak{D}$.

Proof. As usual, let $\mathfrak{U}=\cup_{j \in N} \mathfrak{N}_{A_{j}}$ and $B=B(\mathfrak{D}, \mathfrak{D})=\cup_{j \in \mathbb{N}} \mathscr{N}_{A_{j}}$ where 
$\left(\mathfrak{N}_{A_{j}},\|\|_{A_{j}}\right)$ and $\left(\mathscr{V}_{A_{j}},\|\|_{A_{j}}\right)$ are order-unit normed spaces, with norm \|\|$_{A_{j}}$, induced by the positive element $A_{j}$. One has $B=B^{*}$, and from Corollary 1 , $B$ endowed with bibounded topology is a DF-space. Since $\mathfrak{D} \hat{\otimes} \mathfrak{D}$ is quasinormable, this DF-space is bornological, therefore we get identity on $B$ of the bibounded topology and the $\rho$-topology.

For clarity of the text, let us adopt the following convention: if $E$ is any set endowed with some topology $\mathscr{C}$ and $X$ is a given subset of $E$, notation $(X,(E, \mathscr{C}))$ will refer to the space $X$ endowed with the topology induced by $(E, \mathscr{C})$. Since the space $(B, \rho)$ satisfies the strict condition of Mackey convergence, there exists, for every element $A \geqq 0$ of $\mathfrak{A}$, some element $A_{j}$ such that the identity mapping

$$
\left(\left(-A+B^{+}\right) \cap\left(A-B^{+}\right),\|\|_{A_{j}}\right) \longrightarrow\left(\left(-A+B^{+}\right) \cap\left(A-B^{+}\right),(B, \rho)\right)
$$

is bicontinuous $\left(B^{+}\right.$being the positive cone of $\left.B\right)$. Put $X=\left(-A+B^{+}\right) \cap\left(A-B^{+}\right)$ $\cap \mathfrak{A}=\left(-A+\mathfrak{U}^{+}\right) \cap\left(A-\mathfrak{Q}^{+}\right)$. By restriction to $X$, the spaces $\left(X,\|\|_{A_{s}}\right)$ and $(X,(\mathfrak{B}, \rho))$ are homeomorphic. But $\left(X,\|\|_{A_{j}}\right)$ is finer than $(X,(\mathfrak{A}, \rho))$, which is finer than $(X,(B, \rho))$, since injections from $\mathfrak{N}_{A_{j}}$ into $(\mathfrak{H}, \rho)$ and $(\mathfrak{A}, \rho)$ into $(\mathfrak{B}, \rho)$ are continuous. This establish the proposition, since the assertion relative to the second dual follows from [5] and metrisability of bounded sets.

Proposition 8. Let $\mathfrak{A}$ be as in Theorem 2 with $\mathfrak{D}$ quasi-normable. Denote by $P$ the vector subspace of the strong dual $\mathfrak{A}^{\prime}$ of $(\mathfrak{H}, \rho)$, linearly spanned by linear forms $\omega_{x, y}$ for $x$ and $y$ varying in $\mathfrak{D}$. The following properties holds.

$1^{\circ} P$ is weakly dense in $\mathfrak{I}^{\prime}$.

$2^{\circ}$ if $B$ and $C$ are bounded subsets of $\mathfrak{D}$, the set $\omega_{B, C}$ of elements $\omega_{x, y}$ with $x$ in $B$ and $y$ in $C$, is a bounded subset of $\mathfrak{U}^{\prime}$ included in $P$.

$3^{\circ}$ Every bounded positive subset of $\mathfrak{I}^{\prime}$ is weakly contained in a bounded subset of $P$ of type $\omega_{B, C}$. The positive cone $\mathfrak{A}^{+}$is normal for $\rho$ if and only if every bounded subset of $\mathfrak{Q}^{\prime}$ is weakly contained in a bounded set of type $\omega_{B, C}$.

Proof. In duality $\left\langle\mathfrak{Y}, \mathfrak{P l}^{\prime}\right\rangle$, the polar of $P$ is trivially the null space, therefore our affirmation follows from bipolar theorem. It is easy to see that the sesquilinear map

$$
(x, y) \in \mathfrak{D} \times \mathfrak{D} \longrightarrow \omega_{x, y} \in \mathfrak{U}^{\prime}
$$

is separately continuous, hence continuous in both variables since $\mathfrak{D}$ is a Fréchet space. The image of $B \times C$ is therefore a bounded set in $\mathfrak{I}^{\prime}$, included in $P$.

Introduce now $(\mathfrak{U}, \chi)$ the vector space $\mathfrak{U}$ endowed with the topology of uniform convergence on equicontinuous positive sets of $\mathfrak{U}^{\prime}$ : clearly $\mathfrak{U}^{+}$is normal 
for $\chi$. The bibounded topology corresponds to the uniform convergence of equicontinuous subsets of type $\omega_{B, C}$ and, due to polarization equality, is less finer than $(\mathfrak{A}, \chi)$. Let $V$ be an open disked convex zero neighborhood of $(\mathfrak{A}, \chi)$, order-convex. By induction, we construct a sequence of elements $B_{i} \geqq 0$ of $\mathfrak{U}=\cup_{i} \mathfrak{N}_{A_{i}}$ satisfying for all integer $i B_{i} \leqq B_{i+1}, \mathfrak{N}_{A_{i}}=\mathfrak{N}_{B_{i}}$ and $\left[-B_{i}, B_{i}\right] \subset V$. Thus, the set $\Omega$ which equals to the union of $\left[-B_{i}, B_{i}\right]$ for $i \in \mathbb{N}$, is contained in $V$ and saturated for the order of $\mathfrak{A}$. An adaptation of the proof of Proposition 6 implies the equality of $(\mathfrak{H}, \chi)$ and $\mathfrak{H}$ endowed with the bibounded topology. Therefore the third assertion is a consequence of Mackey theorem.

Theorem 3. Let $\mathfrak{I}$ be a *-algebra satisfying the strict condition of Mackey convergence and $\mathfrak{D}$ be the domain of $\mathfrak{A}$. Assume that every continuous linear form on $\mathfrak{A}$ is $\sigma$-weakly continuous. Then we have the followings.

$1^{\circ}$ The second dual $\mathfrak{A}^{\prime \prime}$ of $\mathfrak{A}$ can be vectorially identified to the $\sigma$-weak closure of $\mathfrak{A}$. This space $\mathfrak{A}^{\prime \prime}$ consists of continuous sesquilinear forms on $\mathfrak{D} \times \mathfrak{D}$, satisfies $\mathfrak{A}^{\prime \prime}=\left(\mathfrak{H}^{\prime \prime}\right)^{*}$, and the order induced by the bipolar of $\mathfrak{A}^{+}$coincides with the order of $B(\mathfrak{D}, \mathfrak{D})$. The strong topology of $\mathfrak{I}^{\prime \prime}$ is exactly the $\rho$-topology for which $\mathfrak{Q}^{\prime \prime}$ is a complete ultrabornological DF-space.

$2^{\circ}$ The second dual $\mathfrak{P}^{\prime \prime}$ is a *-algebra if and only if $\lambda$ agrees with $\rho$ on $\mathfrak{A}$. When it is the case, elements of $\mathfrak{P I}^{\prime \prime}$ are closeable operators preserving the domain $\mathfrak{D}$ invariant.

It should be pointed out that the first assertion of Theorem 3 holds if $\mathscr{A}$ is any subspace of $B(\mathfrak{D}, \mathfrak{D})$ with $\mathfrak{U}=\mathfrak{V}^{*}$, containing a cofinal subset of the positive cone of $B(\mathfrak{D}, \mathfrak{D})$ and satisfying the strict condition of Mackey convergence.

Before proving this theorem, let us carry out the case of a general *-algebra. One has naturally to introduce the universal representation $\pi$ of $\mathfrak{A}$, the direct sum of all G.N.S. representations associated to positive states on $\mathfrak{A}$. To be more precise in details, if $\varphi$ is a linear positive form on $\mathfrak{A}=\cup_{i} \mathfrak{N}_{A_{i}}, \varphi$ canonically defines a Hilbert space $H_{\varphi}$, a dense subspace $\mathfrak{D}_{\varphi}$ of $H_{\varphi}$, and a representation $\pi_{\varphi}$ of $\mathfrak{A}$ such that for $T \in \mathfrak{A}, \pi_{\varphi}(T) \mathfrak{D}_{\varphi} \subset \mathfrak{D}_{\varphi}, \varphi(T)=\left(\pi_{\varphi}(T) \zeta_{\varphi} \zeta_{\varphi}\right)$, with $j_{\varphi}$ the canonical surjection of $\mathfrak{A}$ onto $\mathfrak{D}_{\varphi}$. If $T$ is positive in $\mathfrak{U}$, one has $\pi_{\varphi}(T) \geqq 0$ since

$$
\left(\pi_{\varphi}(T) j_{\varphi}(S), j_{\varphi}(S)\right)=f\left(S^{*} T S\right) \geqq 0
$$

for all $S$ in $\mathfrak{A}$. From [10] it follows that $\pi_{\varphi}$ is a positive homomorphism, therefore the relation $\|T\|_{A_{i}} \leqq 1$ implies $\left\|\pi_{\varphi}(T)\right\|_{\pi_{\varphi}\left(A_{i}\right)} \leqq 1$ so that $\pi_{\varphi}$ defines, 
by restriction, a continuous linear map of norm $\leqq 1$ of the normed space $\left(\mathfrak{N}_{A_{i}},\|\|_{A_{i}}\right)$ into the normed space $\left(\left(\pi_{\varphi}(\mathfrak{H})\right)_{\pi_{\varphi}\left(A_{i}\right)},\|\|_{\pi_{\varphi}\left(A_{i}\right)}\right)$, where $\pi_{\varphi}(\mathfrak{H})_{\pi_{\varphi}\left(A_{i}\right)}$ is the vector subspace of $\pi_{\varphi}(\mathfrak{H})$ consisting of operators bounded above by some homothetic of $\pi_{\varphi}\left(A_{i}\right)$. Hence, $\pi_{\varphi}(\mathfrak{U})$ is a countably dominated *-algebra and $\pi_{\varphi}$ is a continuous homomorphism from $(\mathfrak{H}, \rho)$ into $\left(\pi_{\varphi}(\mathfrak{U}), \rho\right)$. Recall that for $S \in \pi_{\varphi}(\mathfrak{U})$.

$$
\|S\|_{\Pi_{\varphi}\left(A_{i}\right)}=\sup _{x \in \mathcal{D}_{\varphi}} \frac{|(S x, x)|}{\left(\Pi_{\varphi}\left(A_{i}\right) x, x\right)}=\sup _{x \in \hat{\mathfrak{V}}_{\varphi}} \frac{|(S x, x)|}{\left(\Pi_{\varphi}\left(A_{i}\right) x, x\right)},
$$

where $\hat{\mathfrak{D}}_{\varphi}$ is the completion of the metric space.

In particular, in this universal representation $\pi$ of $\mathfrak{A}$, the algebra $\pi(\mathfrak{U})=$ $\oplus_{\varphi \geqq 0} \pi_{\varphi}(\mathfrak{I})$ is countably dominated and one can choose as an invariant domain $D$ the direct sum of $\mathfrak{D}_{\varphi}$ (for $\varphi \in \mathfrak{U}^{\prime+}$ ) or its completion.

Corollary 2. Let $\mathfrak{A}$ be a *-algebra satisfying the strict condition of Mackey convergence and $\pi$ be the universal representation of $\mathfrak{A}$. Denote $\mathfrak{B}=\pi(\mathfrak{U})$ the $*$-algebra, endowed with $\rho$-topology, isomorphic to $(\mathfrak{U}, \rho)$. Then, if the positive cone of $\mathfrak{A}$ is weakly normal, the space $\mathfrak{B}$ satisfies hypothesis and properties of the preceeding theorem: in particular, the $\sigma$-weak closure $\overline{\mathfrak{B}}$ of $\mathfrak{B}$ is isomorphic, under the bitransposed map ${ }^{t} \pi$ of $\pi$, to the second dual $\mathfrak{U}^{\prime \prime}$ of $\mathfrak{Y}$ : the second dual $\mathfrak{I}^{\prime \prime}$ (or $\left.\overline{\mathfrak{B}}\right)$ is a *-algebra if and only if $\lambda$ agrees with $\rho$ on $\mathfrak{A}$. Topology $\rho$ and the bibounded topology agree on $\mathfrak{B}$ (or on $\mathfrak{B}^{\prime \prime}$ ) if and only if the positive cone of $\mathfrak{A}$ is normal for $\rho$.

Proof. We first show Theorem 3 with, as usual, $D$ equals to its completion $\hat{\mathfrak{D}}$. The bilinear map $j$ from $\mathfrak{D} \times \mathfrak{D}$ into $\mathfrak{U}^{\prime}$ defined by

$$
j(x, y)=\omega_{x, y}
$$

is continuous and induces a continuous linear map $j$ from $\mathfrak{D} \hat{\otimes} \mathfrak{D}$ into $\mathfrak{A}^{\prime}$ satisfying $j(x \otimes y)=\omega_{x, y} . \quad$ From hypothesis, this application is surjective. Therefore $\mathfrak{U}^{\prime}$ is isomorphic to some quotient of $\mathfrak{D} \hat{\otimes} \mathfrak{D}$, so that the second dual $\mathfrak{U}^{\prime \prime}$ is vectorially isomorphic to the orthogonal of $j^{-1}(0)$ in $(\mathfrak{D} \otimes \vec{Q})^{\prime}$, exactly equals to bipolar of $\mathfrak{A}$ in duality $\left\langle\mathfrak{D} \hat{\otimes} \mathfrak{D},(\mathfrak{D} \hat{\otimes} \mathfrak{D})^{\prime}\right\rangle$. Since a fundamental system of bounded subsets of $\mathfrak{Q}^{\prime \prime}$ consist of order intervals $\left[-A_{i}, A_{i}\right]$, where $\left(A_{i}\right)_{i \in \boldsymbol{N}}$ is a cofinal subset of the positive cone of $\mathfrak{A}$ and these sets being $\sigma$-weakly compact, we easily deduce the first assertion using [5].

Assume now that topologies $\lambda$ and $\rho$ agree on $\mathfrak{A}=\cup_{i} \mathfrak{N}_{A_{i}}$. The topology of the strong dual of $(\mathfrak{U}, \rho)$ is therefore defined by semi-norms \|\|$\|_{A_{i}}$ for $i \in \boldsymbol{N}$, with 


$$
\|f\|_{A_{i}}=\sup \left\{|f(T)| ; \sup _{x \in \mathcal{D}} \frac{\|T x\|}{\left\|A_{i} x\right\|} \leqq 1\right\}
$$

for $f \in \mathfrak{Q}^{\prime}$. In particular, we get for $x$ in $\mathfrak{D}$ and $y$ in $H$

$$
\left\|\omega_{x, y}\right\| \leqq\left\|A_{i} x\right\|\|y\| .
$$

If $T$ belongs to $\mathfrak{A}^{\prime \prime}$, there exists $M<+\infty$ and $i \in \mathbb{N}$ such that

$$
|T(f)| \leqq M\|f\|_{A_{i}} \quad \text { for } f \in \mathfrak{Q}^{\prime},
$$

hence

$$
\left|T\left(\omega_{x, y}\right)\right| \leqq M\left\|A_{i} x\right\|\|y\| .
$$

Thus there exists a bounded operator $B$ acting in the Hilbert space $H$ such that

$$
T\left(\omega_{x, y}\right)=\left(B A_{i} x, y\right) \quad \text { for } \quad x \in \mathfrak{D}, \quad y \in H
$$

Let $T^{\prime \prime}$ be the closeable operator, defined on $\mathfrak{D}$ by $T^{\prime \prime}=B A_{i}$ : clearly $T^{\prime \prime}$ does not depend on $i$. One has now to show that $T^{\prime \prime}(\mathfrak{D}) \subset \mathfrak{D}$. Let us fix $x$ in $\mathfrak{D}$. The $\operatorname{map} j_{x}$

$$
T \in \mathfrak{A} \longrightarrow j_{x}(T)=T x \in \mathfrak{D}
$$

is continuous when $D$ is endowed with its Fréchet topology. Indeed, it is continuous from $\mathfrak{A}$ into the Hilbert space $H$ and our affirmation follows from

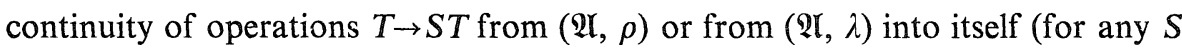
of $\mathfrak{A})$. Since $\mathfrak{D}$ is reflexive, the bitransposed ${ }^{t t} j_{x}$ is a strongly continuous linear map from $\mathfrak{U}^{\prime \prime}$ into $\mathfrak{D}^{\prime \prime}=\mathfrak{D}$. Let us first prove that

$$
{ }^{t} j_{x}(T)=T^{\prime \prime} x
$$

for $T \in \mathfrak{Q} I^{\prime \prime}, T^{\prime \prime}$ being the closeable operator canonically associated to $T$ defined by

$$
T\left(\omega_{x, y}\right)=\left(T^{\prime \prime} x, y\right), \quad x \in \mathfrak{D}, y \in H .
$$

The map

$$
A \in \mathfrak{U} \longrightarrow\left(A_{i} A x, y\right)
$$

for $x \in \mathfrak{D}, y \in H$ is a linear continuous form on $\mathfrak{A}$ (denoted $\omega_{A_{i} \cdot x, y}$ ) and satisfies for $T \in \mathfrak{Q}^{\prime \prime}$

$$
T\left(\omega_{A_{i} \cdot x, y}\right)=\left(\bar{A}_{i}^{*} T^{\prime \prime} x, y\right),
$$

$\bar{A}_{i}^{*}$ being the closed operator equals to the adjoint of the restriction of $A_{i}$ to $\mathfrak{D}$. In fact, we note that the map $y \rightarrow \omega_{A_{i^{\prime}} \cdot x, y}$ is continuous from the Hilbert space $H$ into $\mathfrak{U}^{\prime}$, being obtained from the succession of the continuous map $y \rightarrow \omega_{x, y}$ from $H$ into $\mathfrak{U}^{\prime}$, and the continuous map of $\mathfrak{U}^{\prime}$ into $\mathfrak{U}^{\prime}$ obtained from trans- 
position of the operation $T \rightarrow A_{i} T$ in $\mathfrak{A}$. It follows that relation $(\beta)$ will be true if it is satisfied with $y$ in $\mathfrak{D}$. But, $y$ in $\mathfrak{D}$ implies $\omega_{A_{i} \cdot x, y}=\omega_{x, A_{i} y}$, therefore

$$
\left|T\left(\omega_{A_{i} \cdot x, y}\right)\right|=\left|\left(T^{\prime \prime} x, A_{i} y\right)\right| \leqq M\left\|\omega_{A_{i} \cdot x, y} \mid\right\|_{A_{i}} \leqq M^{\prime}\|y\|
$$

for some suitable constant $M^{\prime}$ depending on $x$ : hence $T^{\prime \prime} x$ belongs to the domain of $\bar{A}_{i}^{*}$ and

$$
T\left(\omega_{A_{i} \cdot x, y}\right)=\left(T^{\prime \prime} x, A_{i} y\right)=\left(\bar{A}_{i}^{*} T^{\prime \prime} x, y\right)
$$

establishes relation $(\beta)$.

Recall that every linear continuous form $f$ on $\mathfrak{D}$ can be written $f(x)=$ ( $A_{i} x, y$ ) with $A_{i}$ and $y$ correctly choosen (not unique) in $\mathfrak{U}$ and $H$, and to simplify notations we denote $f=\left(A_{i} \cdot, y\right)$

The transposed map ${ }^{t} j_{x}$ of $j_{x}$ is the map from $\mathfrak{A}^{\prime}$ into $\mathfrak{D}^{\prime}$ given in duality $\left\langle\mathfrak{D}, \mathfrak{D}^{\prime}\right\rangle$ by

$$
\left\langle j_{x}(A),\left(A_{i}, y\right)\right\rangle=\left\langle A,{ }^{t} j_{x}\left(A_{i}, y\right)\right\rangle=\left\langle A, \omega_{A_{i} \cdot x, y}\right\rangle
$$

for $A$ in $\mathfrak{A}$, so that ${ }^{t} j_{x}$ is defined by

$$
\left({ }^{t t} j_{x}\right)(T)\left(A_{i}, y\right)=T\left(\omega_{A_{i} \cdot x, y}\right)=\left(\bar{A}_{i}^{*} T x, y\right)
$$

with $\left(A_{i}, y\right)$ varying in $\mathfrak{D}^{\prime}$ and $T$ in $\mathfrak{A}^{\prime \prime}$. But the application

$$
f=\left(A_{i} \cdot, y\right) \longrightarrow\left(\bar{A}_{i}^{*} T x, y\right) \in \boldsymbol{C}
$$

defines an element of $\mathfrak{D}^{\prime \prime}$ and, from reflexivity, is associated to a unique vector of $\mathfrak{D}$. Since elements $\left(A_{i} \cdot, y\right)$ with $y$ in $\mathfrak{D}$ and $A_{i}$ in $\mathfrak{A}$ are strongly dense in $\mathfrak{D}^{\prime}$, our vector is well determined by restriction of the preceeding map to this dense subspace, thus coincide with $T^{\prime \prime} x$, proving relation $(\alpha)$. The positive cone $\mathfrak{U}^{\prime+}$ of the dual $\mathfrak{U}^{\prime}$ being normal, every element of $\mathfrak{U}^{\prime \prime}$ is a linear combination of positive ones. Relation $\left(T_{1}+i T_{2}\right)^{*}=T_{1}-i T_{2}$ for $T_{1}=T_{1}^{*}$ and $T_{2}=T_{2}^{*}$ in $\mathfrak{U}$ easily extends to $\mathfrak{I}^{\prime \prime}$. And, from relation $(\alpha)$, we get that elements of $\mathfrak{A}^{\prime \prime}$ and their adjoints send the domain $\mathfrak{D}$ into itself.

It remains to show that $\mathfrak{I}^{\prime \prime}$ is a $*$-algebra. Let $S$ be in $\mathfrak{P}$ : by transposing two times, we see that the operation

$$
\pi(S): T \in \mathfrak{A} \longrightarrow \pi(S) T=S T
$$

extends in

$$
{ }^{t t} \pi(S): T^{\prime \prime} \in \mathfrak{U}^{\prime \prime} \longrightarrow S T^{\prime \prime} \in \mathfrak{U}^{\prime \prime},
$$

since ${ }^{t} \pi(S)$ is completely defined by 
implying

$$
{ }^{t} \pi(S)\left(\omega_{x, y}\right)=\omega_{x, S^{*} y}, \quad x, y \in \mathfrak{D}
$$

$$
\left({ }^{t t} \pi(S) T^{\prime \prime}\right)\left(\omega_{x, y}\right)=T^{\prime \prime}\left(\omega_{x, S^{*} y}\right)=\left(T^{\prime \prime} x, S^{*} y\right)=\left(S T^{\prime \prime} x, y\right) .
$$

In order to extend operation $S \in \mathfrak{U} \rightarrow S T^{\prime \prime} \in \mathfrak{U}^{\prime \prime}$ with $T^{\prime \prime}$ fixed in $\mathfrak{U}^{\prime \prime}$, we need to endow $\mathfrak{U}^{\prime \prime}$ with the weak topology $\sigma\left(\mathfrak{Q}^{\prime \prime}, \mathfrak{P}^{\prime}\right)$ : bounded subsets of $\mathfrak{H}^{\prime \prime}$ for this topology agree with bounded subsets of the strong dual $\mathfrak{U}^{\prime \prime}$ since $\mathfrak{U}^{\prime}$ is Fréchet, and are weakly relatively compact subsets. The map

$$
\delta\left(T^{\prime \prime}\right): S \in \mathfrak{A} \longrightarrow \delta\left(T^{\prime \prime}\right) S=S T^{\prime \prime} \in \mathfrak{U}^{\prime \prime}
$$

is continuous from $(\mathfrak{U}, \rho)$ into the weak dual $\mathfrak{U}^{\prime \prime}$ : indeed, any $f$ in $\mathfrak{A}^{\prime}$ can be written as $f=\sum_{i=1}^{\infty} \omega_{x_{i}, y_{i}}$ with $\left(x_{i}\right)_{i \in \boldsymbol{N}}$ and $\left(y_{i}\right)_{i \in \boldsymbol{N}}$ being $\sigma$-convergent sequences in $D$, so that the map

$$
f \circ \delta\left(T^{\prime \prime}\right): S \in \mathfrak{U} \longrightarrow f\left(S T^{\prime \prime}\right)
$$

can be detailed as

$$
f\left(S T^{\prime \prime}\right)=\sum_{i=1}^{\infty}\left(S T^{\prime \prime} x_{i}, y_{i}\right)
$$

and, from polarization equality, it follows that this linear form is a linear combination of positive ones, therefore is continuous. Thus, the second transposed ${ }^{t t} \delta\left(T^{\prime \prime}\right)$ of $\delta\left(T^{\prime \prime}\right)$ is a continuous linear map from the strong bidual $\left(\mathfrak{P}^{\prime \prime}, \rho\right.$ ) into the weak dual $\mathfrak{A}^{\prime \prime}$. One has for $x$ and $y$ in $\mathfrak{D}$

$$
\left({ }^{t} \delta\left(T^{\prime \prime}\right)\right) \omega_{x, y}=\omega_{T^{\prime \prime} x, y},
$$

the formula being analogous for $f=\sum_{i=1}^{\infty} \omega_{x_{i}, y_{i}}$, hence for $S$ in $\mathfrak{I}^{\prime \prime}$

$$
\left[{ }^{t t} \delta\left(T^{\prime \prime}\right)\right]\left(S^{\prime \prime}\right)\left(\omega_{x, y}\right)=S^{\prime \prime}\left[{ }^{t} \delta\left(T^{\prime \prime}\right)\right]\left(\omega_{x, y}\right)=S^{\prime \prime}\left(\omega_{T^{\prime \prime} x, y}\right)=\left(S^{\prime \prime} T^{\prime \prime} x, y\right)
$$

having here identified operator $S^{\prime \prime}$ with the linear form, it defines on $\mathfrak{A}^{\prime}$.

In short, the multiplication $(S, T) \in \mathfrak{Q} \times \mathfrak{U} \rightarrow S T \in \mathfrak{A}$ extends in $\left(S^{\prime \prime}, T^{\prime \prime}\right)$ $\in \mathfrak{U}^{\prime \prime} \times \mathfrak{U}^{\prime \prime} \rightarrow S^{\prime \prime} T^{\prime \prime} \in \mathfrak{U}^{\prime \prime}$. Since $\mathfrak{U}^{\prime \prime}$ is bornological, this last map is separately continuous hence continuous for both variables showing Theorem 3 .

The proof of Corollary 2 is therefore straightforward. Moreover, when $\mathfrak{A}^{+}$(or equivalently $\mathfrak{B}^{+}$) is normal for $\rho$, any equicontinuous subsets $M$ of $\mathfrak{B}^{\prime}$ corresponds to some bounded subset of the domain. To be more precise, assuming without loss of generality $M$ included in $\mathfrak{B}^{\prime+}$, we associated to each $f_{\alpha}$ of $M$ an element $x_{\alpha}$ of the domain such that $f_{\alpha}(T)=\left(T x_{\alpha}, x_{\alpha}\right)$ for all $T$ of $\mathfrak{B}$. It is immediate that the set of $x_{\alpha}$ is a bounded subset we are looking for and, topology of $\mathfrak{B}$ or of $\mathfrak{B}^{\prime \prime}$ being topology of uniform convergence on equicontinuous 
subset of $\mathfrak{B}^{\prime}$, we get equality of $\rho$-topology and bibounded topology. Other details are trivial.

\section{§3. Particular $*$-Algebras}

This part of the work will discuss particular $*$-algebras often met in group theory: namely, those for which the domain $\mathfrak{D}$ is a Schwartz or nuclear space, for its natural Fréchet topology. This situation of course cannot happen in $\mathbb{C}^{*}$-theory, since Hilbert space of infinite dimension never belong to this type of domain.

Proposition 9. Let $\mathfrak{U}$ be a *-algebra or any subspace of $B(\mathfrak{D}, \mathfrak{D})$ stable under involution containing a cofinal subset of the positive cone of $B(\mathfrak{D}, \mathfrak{D})$. When $\mathfrak{D}$ is a Schwartz space for its Fréchet topology, one has:

$1^{\circ}$ The space ( $\left.\mathfrak{H}, \rho\right)$ the strong dual $\mathfrak{U}^{\prime}$ and the bidual $\mathfrak{A}^{\prime \prime}$ are Schwartz spaces. The completion $\hat{\mathfrak{U}}$ of $\mathfrak{A}$ coincide with $\mathfrak{U}^{\prime \prime}$.

$2^{\circ}$ The positive cone $\mathfrak{U}^{+}$of $\mathfrak{A}$ is normal. Topology $\rho$ and the bibounded topology agree on $\mathfrak{U}$.

It is worthwhile to note that $\mathfrak{U}^{\prime}$ and $\mathfrak{U}^{\prime \prime}$ are reflexive spaces, the situation quite opposite to that of $\mathbf{C}^{*}$-algebras.

Proof. The projective tensor product $\mathfrak{D} \hat{\otimes} \mathfrak{D}$ is a Fréchet space and nuclear space, therefore its strong dual is a Schwartz bornological space. If $B$ is any bounded subset of ( $\mathfrak{A}, \rho$ ), with $\mathfrak{A}=\cup_{i} \mathfrak{N}_{A_{i}}, B$ is bounded in $(\mathfrak{D} \otimes \mathfrak{D})^{\prime}=\cup_{i} \mathscr{N}_{A_{i}}$, hence precompact in some normed space $\left(\mathscr{N}_{A_{i}},\|\|_{A_{i}}\right)$, from the strict condition of Mackey convergence. But $\left(\mathfrak{N}_{A_{i}},\|\|_{A_{i}}\right)$ can be identified to a subspace of $\left(\mathscr{N}_{A_{i}},\|\|_{A_{i}}\right)$, so that $B$ is precompact in $(\mathfrak{A}, \rho)$. Since space $(\mathfrak{A}, \rho)$ is quasinormable it is a Schwartz space. From Mackey theorem, the dual $\mathfrak{I}^{\prime \prime}$ of strong $\mathfrak{U}^{\prime}$ can be identified to the subspace of the weak completion of $\mathfrak{A}$ generated by weak closures of bounded subsets of $\mathfrak{A}$. Since we deal with Schwartz spaces, it follows that $\hat{\mathfrak{A}}$ agree with $\mathfrak{A}^{\prime \prime}$. For the second assertions, we refer to [8], or for more details to [1].

Let $\mathfrak{A}$ be a $*$-algebra and $\mathfrak{D}$ be the dense domain invariant under operators of $\mathfrak{A}$. Define

$$
\mathfrak{D}_{*}=\bigcap_{A \in \mathfrak{A}} \operatorname{Dom}\left(\bar{A}^{*}\right)
$$

where $\bar{A}$ is the closure of the closeable operator $A$ defined on $\mathcal{D}$, and $\bar{A}^{*}$ be 
its adjoint which is a closed operator in the Hilbert space completion of $\mathfrak{D}$. Clearly $\mathfrak{D} \subset \mathfrak{D}_{*}$ and it is convenient to introduce the following.

Definition 4. The domain $\mathfrak{D}$ is called symmetric iff $\mathfrak{D}=\mathfrak{D}_{*}$.

Symmetry of the domain does not depend on the algebraic structure of $\mathfrak{A}$, so that our terminology seems more adapted than [10].

Proposition 10. Let $\mathfrak{A}$ be $a *$-algebra and $f$ be a linear positive (resp. continuous) form on (2l, $\rho$ ). Assume $\mathfrak{D}$ is symmetric and is a Schwartz space. Then there exists, in the Hilbert space $H$ an orthonormal system $\left\{\varphi_{i}\right\}_{i \in N}$ of vectors of $\mathfrak{D}$ and real numbers $\lambda_{i} \geqq 0$ (resp. $\lambda_{i}$ complex numbers) such that for every $T$ of $\mathfrak{T}$

$$
f(T)=\sum_{i=0}^{\infty} \lambda_{i}\left(T \varphi_{i}, \varphi_{i}\right),
$$

the preceeding series being absolutely convergent for all $T$.

Note that the converse of the proposition holds: every linear form on $\mathfrak{A}$, of type $T \rightarrow \sum \lambda_{i}\left(T \psi_{i}, \psi_{i}\right)$ (obvious notations), with absolutely converging series for every $T$ of $\mathfrak{l}$, is continuous. Indeed, such a form is a linear combination of positive ones.

More generally, if $B$ is a bounded subset of the strong dual of $\mathfrak{A}=\cup_{i} \mathfrak{N}_{A_{i}}$ the normality of $\mathfrak{Q}^{+}$(from Proposition 9) implies that there exists bounded positive subsets $B_{1}$ and $B_{2}$ of $\mathfrak{Q}^{\prime}$ such that $B \subset B_{1}-B_{2}$. And, from [9], bounded positive sets of $\mathfrak{U}^{\prime}$ consist of sets $\left\{f_{\alpha}\right\}_{\alpha \in J}$ of positive linear forms satisfying

for all $i$ of $N$.

$$
\sup _{\alpha \in J} f_{\alpha}\left(A_{i}\right)<+\infty
$$

Lemma 1. Let $\mathfrak{A}=\cup_{i} \mathfrak{N}_{A_{i}}$ be a *-algebra, containing the set $\mathscr{F}$ of all bounded operators $B$ of finite rank, acting in the Hilbert space $H$, and satisfying $B(\mathfrak{D}) \subset \mathfrak{D}, B^{*}(\mathfrak{D}) \subset \mathfrak{D}$. If $\mathfrak{D}$ is a Schwartz space, the Hilbert space $H$ is separable and any element of $\mathfrak{A}$ can be approximated in some suitable normed space $\mathfrak{N}_{A_{i}}$ by elements of $\mathscr{F} . \quad$ In particular, $\mathscr{F}$ is dense in $(\mathfrak{U}, \rho)$ for $\rho$-topology.

Note that even the identity operator is limit of elements of $\mathscr{F}$ for $\rho$-topology.

Proof of the lemma. Since $D$ is Fréchet-Montel, $D$ is separable [3], hence contains a countable dense subset. Noting that the norm of the Hilbert space $H$ induces a continuous norm on $\mathfrak{D}$, we get separability of $H$.

Let $\psi_{1}, \ldots, \psi_{n}, \ldots$ be an orthonormal basis of $H$ consisting of elements of D. Let $B_{n}$ be the finite rank operator defined by 


$$
B_{n}(x)=\sum_{j=1}^{n}\left(x, \psi_{j}\right) \psi_{j}, \quad x \in \mathfrak{D} .
$$

Let $A$ be in $\mathfrak{A}$ and $A_{n}=A \cdot B_{n}$. One has, for every $x$ of $\mathfrak{D}$

$$
\lim _{n \rightarrow \infty}\left\|B_{n} x-x\right\|=0 \text {. }
$$

Elements $\left(B_{n}-1\right)$ can be viewed as a linear continuous map from $\mathfrak{D}$ into $H$ and the preceeding relation means that the sequence $\left(B_{n}-I d\right)$ is simply convergent on $\mathfrak{D}$, hence uniformly convergent on any compact subset of $\mathfrak{D}$, or any bounded subset of $\mathfrak{D}$, since $\mathfrak{D}$ is Montel. Linear forms

$$
x \in \mathfrak{D} \longrightarrow\left(\left(B_{n}-1\right) x, y\right)
$$

for $y$ varying in the unit ball of $H$ and $n$ varying in the set of integers, tends uniformly to zero in the strong dual $\mathfrak{D}^{\prime}$ of $\mathfrak{D}$, and quasinormability of $\mathfrak{D}$ shows that $\left(B_{n}-1\right)$ tends to zero in some normed space $\mathfrak{M}_{\Delta}$ ([8]). Namely, we can find $\Delta$ in $\mathfrak{A}$ and a sequence $\varepsilon(n)$ of reals numbers converging to zero satisfying

$$
\left\|\left(B_{n}-1\right) x\right\| \leqq \varepsilon(n)\|\Delta x\|
$$

for all $x$ of $D$. Now this leads to

$$
\left|\left(A-A_{n} x, x\right)\right|=\left|\left(\left(1-B_{n}\right), A^{*} x\right)\right| \leqq \varepsilon(n)\left\|A^{*} x\right\|\|\Delta x\|
$$

showing so approximation of $A$ by the $A_{n}$ in the normed space $\mathfrak{N}_{\boldsymbol{A} A^{*}+\Delta^{*} \boldsymbol{\Delta}}$. One immediately deduces the density of $\mathscr{F}$ in $(\mathfrak{A}, \rho)$.

Proof of the proposition. The $*$-algebra $\mathfrak{A}$ can be written as $\mathfrak{A}=\bigcup_{k \in N} \mathfrak{N}_{A_{k}^{*} A_{k}}$ with for every integer $k$ and $x$ of $\mathfrak{D},\left\|A_{k} x\right\| \geqq\|x\|$ and $A_{k} \geqq 0$. Denote $\mathfrak{I}(\mathfrak{D})$ the $*$-algebra of all operators, bounded or not, which together with their adjoint are defined on $\mathfrak{D}$, and let this domain invariant under their action. With natural notations, one has $\mathfrak{L}(\mathfrak{D})=\cup_{k \in N} \mathscr{N}_{A_{k}^{*} A_{k}}$. Assume $f$ is a linear positive form on $\mathfrak{Q}$, and choose $\tilde{f}$ a positive linear form on $\mathfrak{L}(\mathfrak{D})$, extending $f$, from [8] Proposition 1. The restriction of $\tilde{f}$ to each normed space $\mathscr{N}_{A_{k}^{*} A_{k}}$ being continuous, there exists, for every integer $k$, a finite number $M_{k}$ such that

$$
|f(T)| \leqq M_{k}\|T\|_{A_{k}^{*} A_{k}}
$$

for $T$ in $\mathscr{N}_{A_{k}^{*} A_{k}}^{*}$. Denote, for $\varphi$ and $\psi$ in $\mathfrak{D}$, by $(\cdot, \varphi) \psi$, the rank one operator associated to the one-dimensional projector $x \in \mathfrak{D} \rightarrow(x, \varphi) \psi$. One has

$$
\left|f\left(\left(\cdot, A_{k} \varphi\right) \psi\right)\right| \leqq M_{k}\|\varphi\|\|\psi\|,
$$

thus we get a bounded positive operator $t$, acting in the Hilbert space $H$, with property

$$
f((\cdot, \varphi) \psi)=(t \psi, \varphi)
$$


Since $(\cdot, \varphi) \varphi$ is positive in $\mathfrak{L}(\mathfrak{D}), t$ is hermitian and the relation

$$
\left|\left(1 \psi, A_{k} \varphi\right)\right| \leqq M_{k}\|\varphi\|\|\psi\|
$$

implies $t^{*}(H)=t(H) \subset \cap_{k \in \boldsymbol{N}} \operatorname{Dom}\left(\bar{A}_{k}^{*}\right)=D_{*}=\mathfrak{D}$. As $D$ is a Schwartz space, the injection from $\mathfrak{D}$ into $H$ is a linear compact map. From closed graph theorem, $t$ is continuous from the Hilbert space $H$ into the Fréchet space D, implying that $t$ is a compact operator in the Hilbert space $H$. Let $\left\{\varphi_{i}\right\}_{i \in \boldsymbol{N}}$ be an orthonormal basis (necessarily countable) associated to the spectral decomposition of $t$. One has $t \varphi_{i}=\lambda_{i} \varphi_{i}$ from some suitable $\lambda_{i} \geqq 0$. Therefore, the relation $t(H) \subset \mathfrak{D}$ implies that $\varphi_{i} \in \mathfrak{D}$ for $\lambda_{i}>0$.

Let us now show that, for every $A$ in $\mathfrak{E}(\mathfrak{D})$, the series $\sum_{n=0}^{\infty} \lambda_{n}\left(A \varphi_{n}, \varphi_{n}\right)$ converges absolutely. The element $A$ belonging to some $\mathscr{N}_{A_{k}}$ or $\mathscr{N}_{A_{k} A_{k}}^{*}$, we can assume $A$ equal to $A_{k}$. Put for $n$ and $k$ in $\mathbb{N}$,

$$
A_{k}^{(n)}=\sum_{i=0}^{n}\left(\cdot, A_{k} \varphi_{i}\right) \varphi_{i} .
$$

Clearly $A_{k}^{(n)}$ belongs to $\mathfrak{L}(\mathfrak{D})$ and

$$
\sup _{x \in \mathfrak{D}} \frac{\left|\left(A_{k}^{(n)} x, x\right)\right|}{\left(A_{k} A_{k} x, x\right)} \leqq \frac{1}{2} \sup _{x \in \mathscr{D}} \frac{\left\|A_{k} x\right\|^{2}+\|x\|^{2}}{\left\|A_{k} x\right\|^{2}} \leqq 1
$$

gives $\left|\tilde{f}\left(A_{n}\right)\right| \leqq M_{k}$ : but

$$
\tilde{f}\left(\left(\cdot, A_{k} \varphi_{i}\right) \varphi_{i}\right)=\left(t \varphi_{i}, A_{k} \varphi_{i}\right)=\lambda_{i}\left(A_{k} \varphi_{i}, \varphi_{i}\right),
$$

thus

$$
\sum_{i=0}^{n} \lambda_{i}\left(A_{k} \varphi_{i}, \varphi_{i}\right) \leqq M_{k}
$$

From positivity of terms of this series, we deduce the convergence, and the correspondence

$$
A \in \mathfrak{L}(\mathfrak{D}) \longrightarrow \sum_{n=0}^{\infty} \lambda_{n}\left(A \varphi_{n}, \varphi_{n}\right)
$$

is clearly a positive linear form, which agree with $f$ on operators of rank one, therefore on $\mathfrak{L}(\mathfrak{D})$ by Lemma 1 and [8] Theorem 1 .

Let us now turn to the case that $f$ continuous on $(\mathfrak{H}, \rho)$. We can assume $f$ is hermitian on $\mathfrak{A}$, and from Hahn-Banach theorem, $f$ can be extended to an hermitian linear continuous form on $(\mathfrak{L}(\mathfrak{D}), \rho)$ : indeed, from Proposition 6, the space $\mathfrak{L}(\mathfrak{D})$ endowed with $\rho$ induces on the subspace $\mathfrak{A}$ the topology $\rho$. An adaptation of preceeding arguments leads to a compact operator $t$ hermitian in the Hilbert space $H$, and absolute convergence of series is obtained by in- 
troducing the $A_{k}^{(n)}$ uniquely with respect to vectors $\varphi_{i}$ associated to scalars $\lambda_{i}$ of same sign.

Corollary 3. Let $\mathfrak{A}$ be a *-algebra with $\mathfrak{D}$ nuclear and $H$ be the Hilbert space completion of $\mathfrak{D}$. Denote $\mathfrak{L}_{b}(H, \mathfrak{D})$ the real vector space of continuous linear map $t$ from the Hilbert space $H$ into the Fréchet space $\mathfrak{D}$ such that $t=t^{*}$, endowed with topology of uniform convergence on the unit ball of $H$. Then we have the followings.

$1^{\circ}$ The space $(\mathfrak{U}, \rho)$ is a nuclear DF-space and its strong dual $\mathfrak{U}^{\prime}$ is a nuclear Fréchet space.

$2^{\circ}$ ) If $\mathfrak{D}$ is symmetric, any hermitian linear continuous linear form $f$ on ( $\mathfrak{H}, \rho$ ) can be written

$$
f(A)=\operatorname{Tr}(A t), \quad A \in \mathfrak{U}
$$

for some suitable $t \in \mathfrak{L}_{b}(H, \mathfrak{D})$ : here $\operatorname{Tr}$ stands for usual trace in $H$. If $f$ is positive, $t$ can be choosen positive. The correspondence $t \in \mathfrak{L}_{b}(H, \mathfrak{D}) \rightarrow f \in \mathfrak{U}^{\prime}$, associated to the preceeding relation is well defined and is a surjective homomorphism of Fréchet spaces exchanging bounded sets.

The proof is left to the reader.

\section{References}

[1] Araki, H. and Jurzak, J. P., On a certain class of $*$-algebras, To appear.

[2] Dixmier, J., Les $\boldsymbol{C}^{*}$-algèbres et leurs representations, Gauthier-Villars, Paris, 1964.

[3] Dieudonne, J., Sur les espaces de Montel métrisables, C. R. Acad. Sc. Paris, 238, (1954), 194-195.

[4] Dieudonne, J., and Gomes, A. P., Sur certains espaces vectoriels topologiques, $C$. R. Acad. Sc. Paris, 230, (1950), 1129-1130.

[ 5 ] Grothendieck, A., Sur les espaces (F) et (DF), Suma Brasil, Marh, 3 (1954), 57-123.

[6] —, Produits tensoriels topologiques et espaces nucléaires, Mem. Amer. Math. Soc., ${ }^{\circ} 16$ (1955).

[7] Jameson, G., Ordered linear spaces, Springer-Verlag, Berlin, 1970.

[ 8 ] Jurzak, J. P., Propriétés topologiques des algèbres d'opérateurs non-bornés, Thèse.

[9] - Simple facts about algebras of unbounded operators, J. Funct. Analysis, 21 (1976), 469-482.

[10] Powers, R. T., Self-adjoint algebras of unbounded operators, Comm. Math. Phys., 21 (1971), 85.

[11] Sherman, T., Positive functionals on *-algebras of unbounded operators, J. Math. Anal. Appl., 22 (1968), 285-318.

[12] Woronowicz, S. L., The quantum problem of moments, II, Reports Math. Phys., 1, (1971) 175-183. 